\title{
A role for the RNA-binding protein MOS2 in microRNA maturation in Arabidopsis
}

\author{
Xueying $\mathrm{Wu}^{1,2}$, Yupeng $\mathrm{Shi}^{2,5}$, Jingrui $\mathrm{Li}^{2,6}$, Le Xu${ }^{4}$, Yuda Fang ${ }^{7}, \mathrm{Xin}^{2} i^{8}$, Yijun $\mathrm{Qi}^{3,4}$ \\ ${ }^{1}$ College of Life Sciences, Beijing Normal University, Beijing 100875, China; ${ }^{2}$ National Institute of Biological Sciences, Zhong- \\ guancun Life Science Park, Beijing 102206, China, ${ }^{3}$ Tsinghua-Peking Center for Life Sciences, Beijing 100084, China; ${ }^{4}$ School of \\ Life Sciences, Tsinghua University, Beijing 100084, China; ${ }^{5}$ Graduate Program, Chinese Academy of Medical Sciences and Peking \\ Union Medical College, Beijing 100730, China; ${ }^{6}$ College of Biological Sciences, China Agricultural University, Beijing 100193, \\ China; ${ }^{7}$ Shanghai Institute of Plant Physiology and Ecology, Chinese Academy of Sciences, Shanghai 200032, China; ${ }^{8}$ Michael \\ Smith Laboratories, University of British Columbia, Vancouver, BC, Canada V6T $1 Z 4$
}

microRNAs (miRNAs) play important roles in the regulation of gene expression. In Arabidopsis, mature miRNAs are processed from primary miRNA transcripts (pri-miRNAs) by nuclear HYL1/SE/DCL1 complexes that form Dicing bodies (D-bodies). Here we report that an RNA-binding protein MOS2 binds to pri-miRNAs and is involved in efficient processing of pri-miRNAs. MOS2 does not interact with HYL1, SE, and DCL1 and is not localized in Dbodies. Interestingly, in the absence of MOS2, the recruitment of pri-miRNAs by HYL1 is greatly reduced and the localization of HYL1 in D-bodies is compromised. These data suggest that MOS2 promotes pri-miRNA processing through facilitating the recruitment of pri-miRNAs by the Dicing complexes.

Keywords: miRNA; RNA binding protein; Arabidopsis; Dicer

Cell Research (2013) 23:645-657. doi:10.1038/cr.2013.23; published online 12 February 2013

\section{Introduction}

MicroRNAs (miRNAs) are key components in the eukaryotic gene regulatory networks [1]. Most miRNAs are transcribed by RNA polymerase II as long primary transcripts, termed as primary miRNAs (pri-miRNAs). The maturation of a miRNA from its pri-miRNA requires two processing steps: 1 ) the pri-miRNA is processed into a stem loop-structured precursor miRNA (pre-miRNA), and 2) the pre-miRNA is in turn processed into a duplex comprising miRNA and miRNA* (the opposite strand that pairs with miRNA). In animals, pri-miRNAs are cleaved into pre-miRNAs by the RNase III Drosha in the nucleus. Pre-miRNAs are exported into the cytoplasm, where they are further cleaved into miRNA/miRNA* duplexes by another RNase III Dicer $[1,2]$. Plants lack

Correspondence: Yijun Qi

Tel: +86-10-62793132; Fax: +86-10-62793792

E-mail: qiyijun@biomed.tsinghua.edu.cn

Received 22 November 2012; revised 2 January 2013; accepted 4 January 2013; published online 12 February 2013
Drosha homologs, and both cleavage steps are carried out in the nucleus by one of the four Dicer-like (DCL) proteins, DCL1 [3-6].

In Arabidopsis, additional factors are required for efficient and accurate maturation of miRNAs. These include CBP20/CBP80 (the two subunits of the nuclear cap-binding complex) [7-9], DAWDLE (DDL) [10], HYPONASTIC LEAVES1 (HYL1) [11, 12], and SERRATE (SE) [13, 14]. Mutations in CBP20 or CBP80 lead to decreased accumulation of miRNAs and increased pri-miRNA levels and it has been hypothesized that CBP20/CBP80 might facilitate the access of pri-miRNAs to the processing machinery [7-9]. DDL is a forkhead-associated (FHA) domain protein that interacts with DCL1. In $d d l$ mutants, both miRNAs and pri-miRNAs are reduced. DDL was proposed to be a candidate protein that recruits DCL1 to pri-miRNAs [10]. HYL1 is a dsRNA-binding protein [11, $12]$, and $\mathrm{SE}$ is a $\mathrm{C} 2 \mathrm{H} 2$ zinc-finger protein $[13,14]$. Both proteins interact with DCL1 and they together form dicing complexes in the nuclear Dicing bodies (D-bodies) $[15,16]$. Biochemical studies have shown that HYL1 and SE promote the efficiency and accuracy of pri-miRNA processing by DCL1 [17]. Recently, it has been shown 
that the activity of HYL1 in miRNA processing is regulated by C-Terminal Domain Phosphatase-Like 1 (CPL1) [18].

After processing, the miRNA/miRNA* duplex is methylated by Hua Enhancer 1 (HEN1) at the 3 '-termini $[19,20]$. The miRNA strand of the duplex is selectively loaded onto ARGONAUTE1 (AGO1) to form miRNAinduced silencing complexes (miRISCs) [21-24]. Biochemical studies using extracts of evacuolated tobacco BY-2 protoplasts have indicated that the loading of miRNAs onto AGO1 is facilitated by HSP90 and Cyclophilin 40 (CYP40) [25-27]. miRNA loading into AGO1 is also negatively regulated by an Importin $\beta$ protein EMA1 [28]. Through base pairing, miRISCs are guided by miRNAs to their target transcripts to mediate target mRNA cleavage or translational repression [29]. In addition to the canonical miRNAs, plants encode a class of DCL3-dependent long miRNAs (lmiRNAs), which are associated with AGO4 and mediate DNA methylation at their target genes [30].

MOS2 (At $\lg 33520)$ was first identified in a genetic screen for mutants that are defective in innate immunity [31]. It encodes a nuclear protein with G-patch and KOW domains that are indicative of RNA binding [31]. In this study, we show that MOS2 is involved in primiRNA processing. MOS2 binds to pri-miRNAs and is required for the recruitment of pri-miRNAs by HYL1 and the localization of HYL1 in D-bodies. Our data suggest that MOS2 promotes pri-miRNA processing through facilitating the recruitment of pri-miRNAs by the Dicing complexes.

\section{Results}

MOS2 is required for the biogenesis of miRNAs, tasiRNAs, and hc-siRNAs

mos 2-2 is a T-DNA insertional mutant allele of MOS2 in the Columbia-0 (Col-0) background (SALK_033856) [31]. The mos 2-2 mutant has reduced stature and displays pleiotropic morphological phenotypes including shorter roots, rounder leaves, and more bushy stems (Figure 1A), reminiscent of the phenotypes of the mutants that are defective in miRNA biogenesis. This prompted us to investigate whether miRNA biogenesis was compromised in $\operatorname{mos} 2-2$. RNA blot analysis showed that the accumulation of all 11 examined miRNAs (miR156, 159, $161,166,167,168,171,172,173,319$, and 393) was significantly reduced in $\operatorname{mos} 2-2$, albeit to a lesser extent than that in the hyl1-2 mutant (Figure 1B). Transgenic expression of C-terminally YFP-tagged MOS2 under the control of its native promoter ( $p M O S 2: \because M O S 2-Y F P$ ) fully complemented the morphological defects (Figure
$1 \mathrm{C})$ and restored the accumulation of miRNAs in mos $2-2$ (Figure 1B), demonstrating that the reduced accumulation of miRNAs in mos2-2 was caused by the loss-offunction of MOS2.

We next investigated whether MOS2 is involved in the production of other endogenous small RNAs including trans-acting siRNAs (ta-siRNAs) and heterochromatic siRNAs (hc-siRNAs) $[29,32]$. We found that the examined ta-siRNA (ta-siR255) and hc-siRNAs (AtREP2, SIMPLEHAT2, and siR 1003) were reduced by $40 \%-60 \%$ in mos 2-2 (Figure 1B), and this reduction was complemented by the MOS2-YFP transgene (Figure 1B).

In order to probe the global effect of MOS2 on gene expression, we performed high throughput RNA sequencing (RNA-seq) analysis. We found that 243 genes were down-regulated by at least 2-fold (Figure 2A and Supplementary information, Table S1) and 94 genes were up-regulated by at least 2-fold in mos2-2 (Figure 2A and Supplementary information, Table S2). Intriguingly, several genes encoding defensins were the most downregulated (Supplementary information, Table S1), which is consistent with the finding that the mos 2 mutant has decreased resistance against pathogens [31]. However, the miRNA target genes were not recovered from the RNA-seq analysis likely because of the limited sequencing depth (Supplementary information, Tables S1 and S2). We thus used quantitative RT-PCR to measure the expression of miRNA target genes in mos 2-2. In negative correlation to the reduced accumulation of miRNAs, the expression levels of 17 examined miRNA targets were significantly increased in mos 2-2 (Figure 2B).

To further establish the role of MOS2 in miRNA biogenesis, we crossed $\operatorname{mos} 2-2$ to hyl1-2, a null allele of HYL1 [12]. We found that the mos 2-2 hyll-2 double mutant showed more severe developmental phenotypes (Figure 3A), reduced accumulation of miRNAs (Figure $3 \mathrm{~B}$ ), and elevated expression of miRNA targets (Figure $3 \mathrm{C}$ ), compared to those in mos $2-2$ or hyll-2 single mutants. We also generated a double mutant of $\operatorname{mos} 2-2$ and ago1-25 [33], a hypomorphic allele of AGO1. Consistent with the more severe developmental phenotypes (Figure 3D), in the mos2-2 ago1-25 double mutant, the expression of miRNA targets was further elevated (Figure 3E), compared to those in the mos $2-2$ or agol-25 single mutant.

Taken together, these observations indicate a general role for MOS2 in small RNA biogenesis.

\section{MOS2 is involved in pri-miRNA processing}

The reduced miRNA accumulation in mos $2-2$ could be attributed to reduced pri-miRNA transcription/stability or decreased efficiency of pri-miRNA processing. 
A
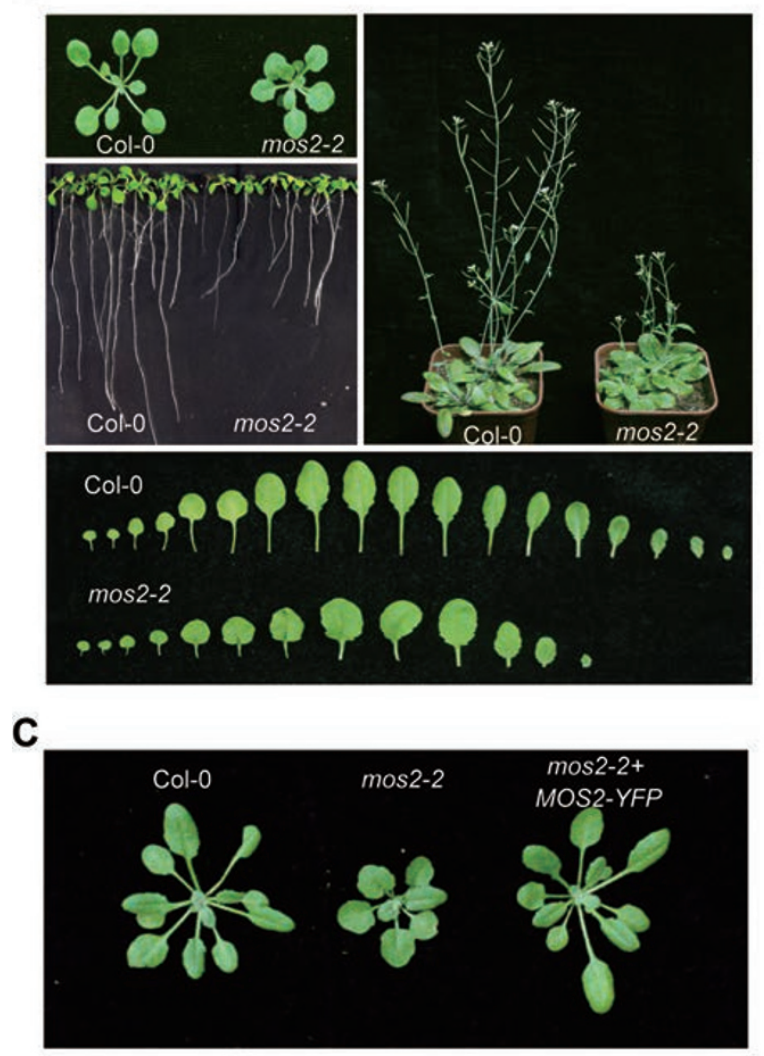

B

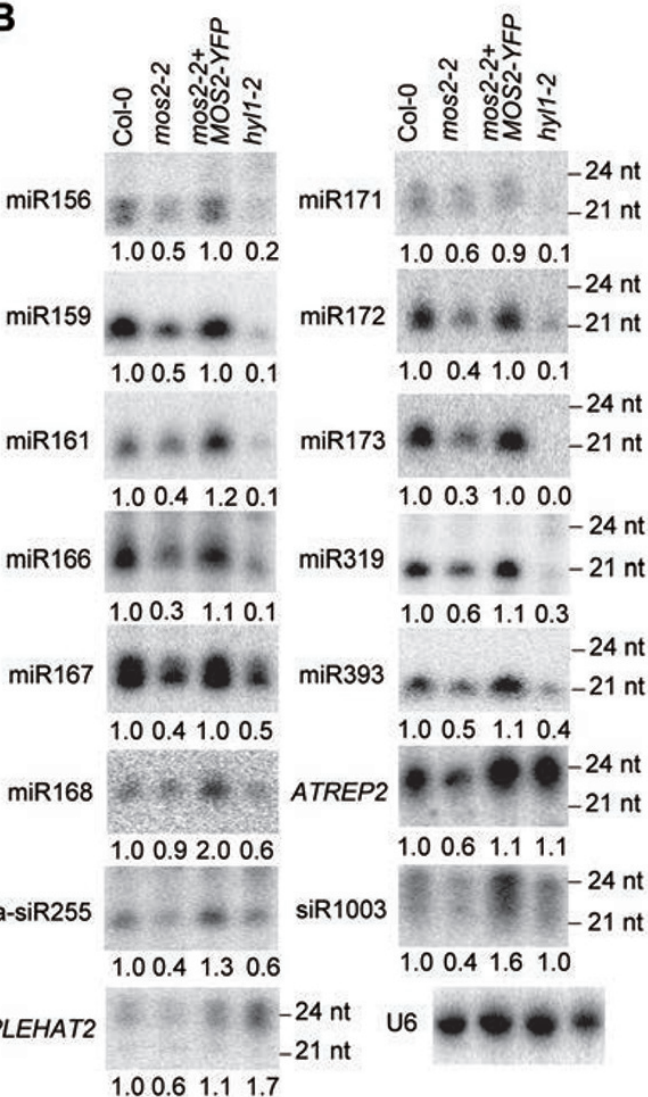

Figure 1 MOS2 is required for the accumulation of miRNAs, ta-siRNAs, and hc-siRNAs. (A) A catalog of photographs of Col-0 and mos2-2 plants at different developmental stages. The plants were grown under long days. (B) Small RNA northern blot analysis of miRNAs, ta-siRNAs, and hc-siRNAs in Col-0, mos2-2, hyl1-2, and a mos2-2 transgenic line containing pMOS2::MOS2-YFP transgene. Small RNAs were extracted from 3-week-old seedlings. U6 was also probed and used as a loading control. The miRNA signals were quantified and normalized to U6 RNAs, and the relative values were calculated by comparison with those in Col-0 (arbitrarily set to 1.0). (C) Photographs of 4-week-old plants with the indicated genotypes.

To test these possibilities, we determined the levels of pri-miRNA transcripts by quantitative RT-PCR (qRTPCR). Five examined pri-miRNAs (pri-miR159a, 159b, $167 a, 171 c$, and $393 a$ ) accumulated to significantly higher levels in the mos2-2 mutant than in the wild type (Col-0) plants (Figure 4A). To rule out the possibility that increased accumulation of pri-miRNAs in $\operatorname{mos} 2-2$ was due to increased transcription of the miRNA genes, we examined the effect of the mos 2-2 mutation on the expression of a GUS reporter gene under the control of MIR $159 b$ or MIR 398c promoter. The transgenic lines pMIR159b::GUS [34] and pMIR398c::GUS [35] were crossed into mos 2-2, respectively. Progenies homozygous for both the transgene and mos2-2 were obtained. No obvious changes in intensity of the GUS staining signal were observed in the mos $2-2$ mutant background, compared to that in the Col-0 background (Figure 4B). We also measured the transcript levels of GUS in these plants by quantitative RT-PCR. In agreement with the GUS staining results, the levels of GUS transcripts were comparable in the Col-0 and mos2-2 backgrounds (Figure 4C).

Taken together, these data indicate that MOS2 unlikely regulates the transcription of miRNA genes and instead is involved in pri-miRNA processing.

\section{MOS2 binds pri-miRNAs both in vitro and in vivo}

The presence of G-patch and KOW domains in MOS2 [31] and its involvement in pri-miRNA processing led us to examine whether MOS2 could bind pri-miRNAs. We first performed RNA electrophoretic mobility shift assay using recombinant MOS2 and in vitro transcribed ${ }^{32} \mathrm{P}$ labeled pri-miR159b (Figure 5A). As shown in Figure 5B (left panel), MOS2 was able to bind pri-miR159b transcripts and caused a gel shift of the hot probe. To confirm the RNA binding affinity of MOS2, we performed com- 

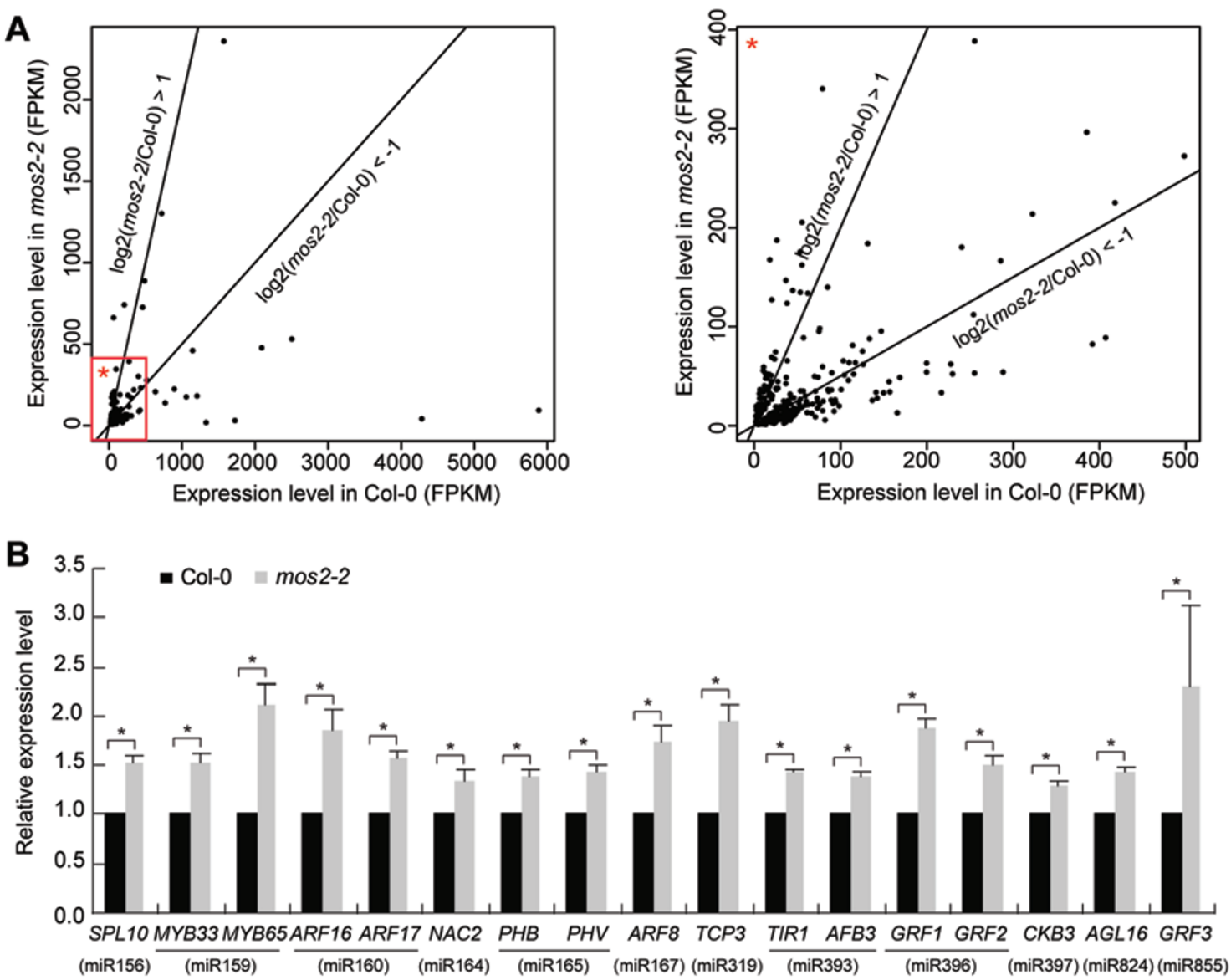

Figure 2 The effect of mos2 mutation on the expression of genes including miRNA targets. (A) Scatter plots of the FPKM of each gene in the Col-0 versus that in the mos2-2 mutant. Average FPKMs from two biological replicates were used to generate the histogram. See Supplementary information, Tables S1 and S2 for lists of genes that were down-regulated or up-regulated at least 2-fold in mos2-2. (B) Detection of the expression levels of several miRNA target genes in 3-week-old seedlings of Col-0 and mos2-2 by quantitative RT-PCR. GAPDH was used as an internal control and for normalization of the data. Error bars indicate $\operatorname{SD}(n=3)$, and asterisks indicate a significant difference between the indicated samples $(t$ test, $P<0.05)$.

petition assays using unlabelled pri-miR159b transcripts. Increasing amounts ( 0.1 to 2 pmols) of unlabeled pri$m i R 159 b$ were added into the binding reaction mixture containing MOS2 and labeled pri-miR159b (0.5 pmol). As shown in Figure 5B (middle panel), the signal indicating bound pri-miR $159 \mathrm{~b}$ decreased proportionally to the amount of unlabelled pri-miR159b added. However, addition of unlabelled GAPDH transcripts in the binding reaction also produced similar results (Figure $5 \mathrm{~B}$, right panel). These data indicate that MOS2 indeed has RNA binding activity, but this activity is not specific to primiRNAs in vitro.

Next we examined whether MOS2 could bind to primiRNAs in vivo. Because we failed to recover sufficient amount of MOS2 protein from the $p M O S 2:: M O S 2-Y F P$ transgenic plants, we generated a transgenic line that expresses N-terminally FLAG-tagged MOS2 (FLAG-
MOS2) under the control of the $35 \mathrm{~S}$ promoter in the mos 2-2 mutant background. The normal biological function of FLAG-MOS2 was evidenced by its ability to complement the developmental defects of mos 2-2 (Supplementary information, Figure S1). FLAG-MOS2 was immunoprecipitated from the mos 2-2+FLAG$M O S 2$ plants after cross-linking (Figure 5C). Eleven primiRNAs including pri-miR159b, 160c, 161, 167d, 170, $171 c, 172 a, 173,393 b, 403$, and 824 were detected in the FLAG-MOS2 immunoprecipitates, but were barely detectable in the control immunoprecipitates (Figure 5C), indicating that MOS2 binds pri-miRNAs in vivo. GAPDH mRNAs were not detected in FLAG-MOS2 immunoprecipitates, suggesting that MOS2 binds pri-miRNAs specifically in vivo. Such binding specificity might be provided by other yet-to-be identified cellular factors. 
A

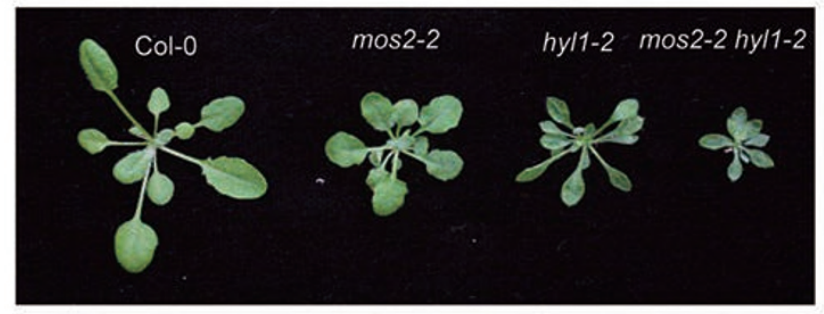

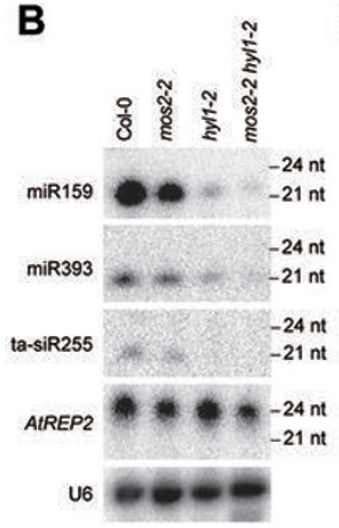

D

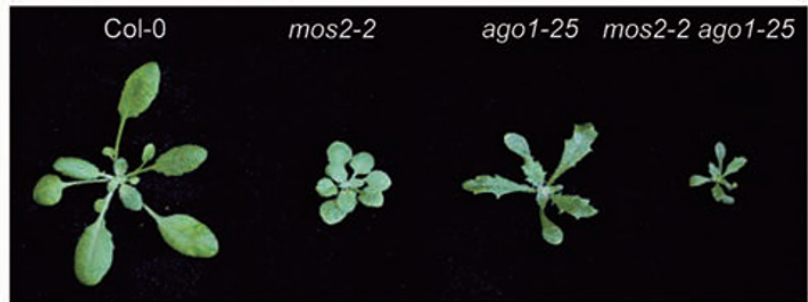

E

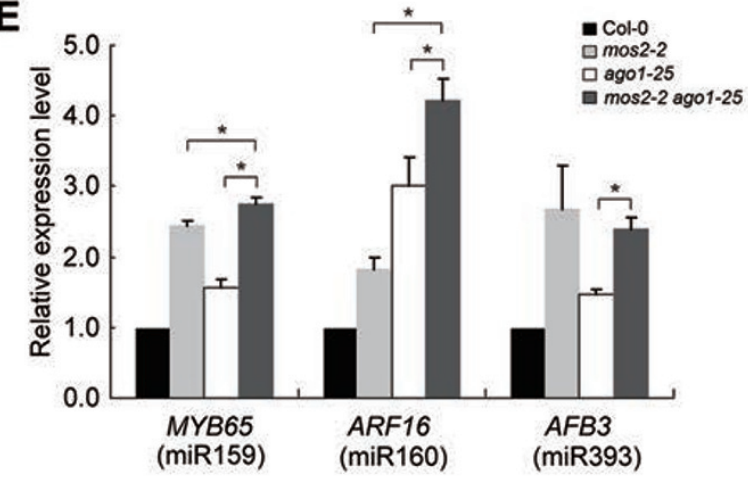

MOS2 does not interact with the known protein components of D-bodies

The binding of MOS2 to pri-miRNAs implicates a direct role for MOS2 in pri-miRNA processing. We thus tested whether MOS2 could interact with the known proteins (DCL1, HYL1, and SE) in D-body $[15,16]$. We first examined whether MOS2 is localized in D-bodies.
Figure 3 Characterization of the mos2-2 hyl1-2 and mos2-2 ago1-25 double mutants. (A) Phenotypes of the mos2-2, hyl1-2, and mos2-2 hyl1-2 mutants. Pictures were taken from 4-weekold plants. (B) Small RNA northern blot analysis of miRNAs in Col-0, mos2-2, hyl1-2, and mos2-2 hyl1-2 plants. Small RNAs were extracted from 3-week-old seedlings. U6 was also probed and used as a loading control. (C) Detection of the expression levels of the indicated miRNA target genes in 3-week-old seedlings of Col-0, mos2-2, hyl1-2, and mos2-2 hyl1-2 plants by quantitative RT-PCR. GAPDH was used as an internal control and for normalization of the data. Error bars indicate SD $(n=3)$ and asterisks indicate a significant difference between the indicated samples ( $t$ test, $P<0.05$ ). (D) Phenotypes of the mos2-2, ago1-25, and mos2-2 ago1-25 mutants. Pictures were taken from 4-week-old plants. (E) Detection of the expression levels of the indicated miRNA target genes in 3-week-old seedlings of Col-0, mos2-2, ago1-25, and mos2-2 ago1-25 by quantitative RT-PCR. GAPDH was used as an internal control and for normalization of the data. Error bars indicate SD $(n=3)$ and asterisks indicate a significant difference between the indicated samples ( $t$ test, $P<0.05$ ).

MOS2-YFP was transiently expressed under the control of its native promoter in Nicotiana benthamiana cells by agroinfiltration. MOS2-YFP was evenly distributed in the nucleus, in contrast to the predominant localization of HYL1-YFP in the nuclear D-bodies when it was expressed under the control of HYL1 native promoter (Figure 6A). Similarly, HYL1-YFP was found in Dbodies in the pHYL1::HYL1-YFP transgenic Arabidopsis as previously reported $[15,16]$, whereas MOS2-YFP was not observed to form any discrete nuclear bodies in the pMOS2::MOS2-YFP transgenic plants (Supplementary information, Figure S2). These data suggest that MOS2 is not localized in D-bodies.

We next investigated whether MOS2 could interact with DCL1, HYL1, and SE. We employed the bimolecular fluorescence complementation (BiFC) approach to examine the interactions between these proteins. Protein partners were fused to $\mathrm{N}$ - or C-terminal fragments of YFP, respectively, and expressed in $N$. benthamiana cells by agroinfiltration. Confirming previous results, $\mathrm{BiFC}$ signals between DCL1, HYL, and SE were observed in D-bodies (Figure 6B). However, no BiFC signal was observed between MOS2 and DCL1 or MOS2 and HYL1 (Figure 6B). Intriguingly, BiFC signal between MOS2 and SE was observed in nuclear foci (Figure 6B). As MOS2 is not localized in the D-body (Figure 6A and Supplementary information, Figure S2), we reasoned that these nuclear foci were probably not D-bodies and might be BiFC artifacts. We further carried out co-immunoprecipitation experiments to test whether MOS2 interacts with DCL1, HYL1, and SE. Initial immunoprecipitation 


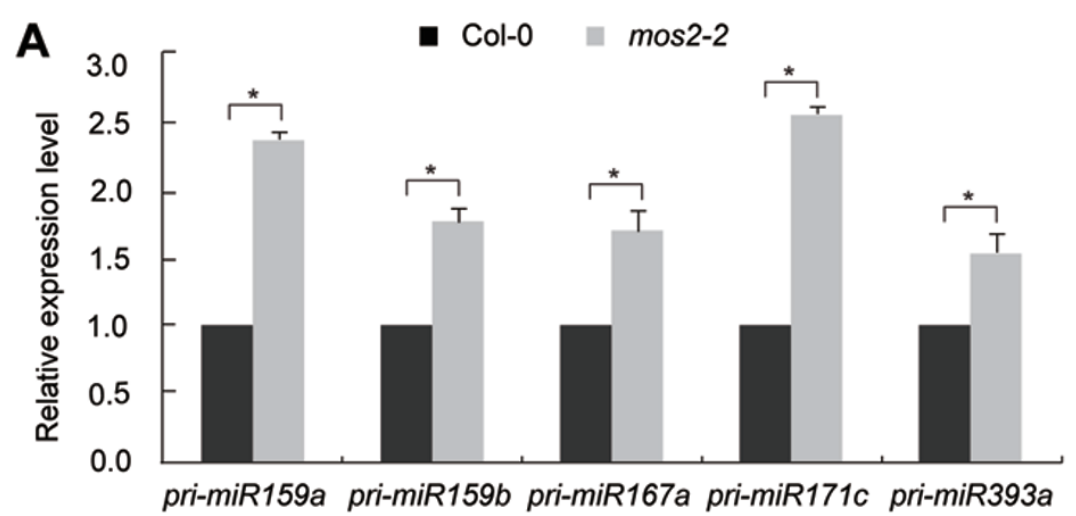

B

pMIR159b::GUS

pMIR398c::GUS
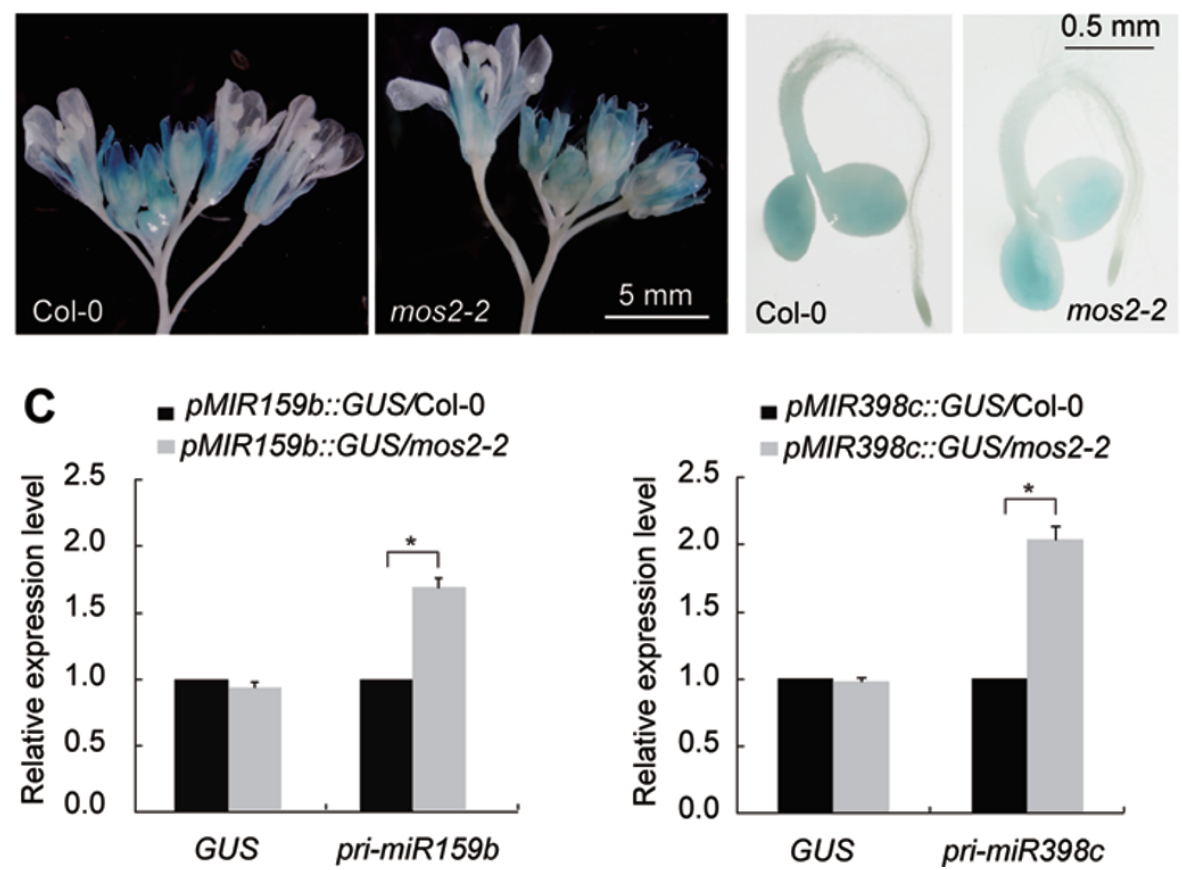

Figure 4 MOS2 is involved in pri-miRNA processing. (A) Detection of the expression levels of the indicated pri-miRNAs in the Col-0 and mos2-2 seedlings by quantitative RT-PCR. Error bars indicate SD $(n=3)$, and asterisks indicate a significant difference between the indicated samples ( $t$ test, $P<0.05$ ). (B) Representative GUS staining images of pMIR159b::GUS (inflorescences), pMIR398c::GUS (5-day-old seedlings) transgenic plants in Col-0 and mos2-2 backgrounds. (C) Quantitative RT-PCR analysis of the transcript levels of GUS and pri-miRNAs in the indicated plants. Error bars indicate SD ( $n=3)$, and asterisks indicate a significant difference between the indicated samples ( $t$ test, $P<0.05)$.

experiments with agroinfiltrated $N$. benthamiana cells produced high background signals, thus we used Arabidopsis protoplast transfection for co-immunoprecipitation experiments. Epitope-tagged MOS2, SE, HYL1, and N-terminal and C-terminal fragments of DCL1 were transiently expressed, and the tagged proteins were immunoprecipitated using epitope-specific antibodies. As shown in Figure 6C, SE, HYL1, and the two DCL1 fragments were not able to pull down MOS2. Furthermore, in yeast two-hybrid experiments, the interaction between
HYL1 and SE was detected, but no interaction was observed between MOS2 and SE (Figure 6D). These observations suggest that MOS2 does not interact with DCL1, HYL1, and SE and is not a component in D-bodies.

MOS2 facilitates the recruitment of pri-miRNAs by HYL1 and the localization of HYL1 in D-bodies

The association of MOS2 with pri-miRNAs but not with the Dicing complex raised a possibility that MOS2 might be involved in pri-miRNA processing by facili- 
A

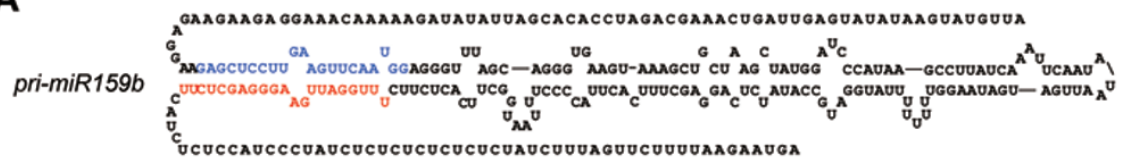

B

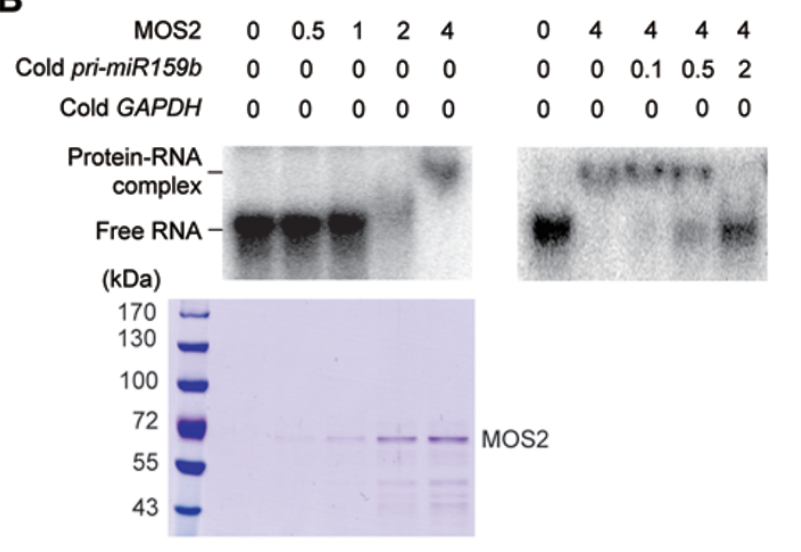

$$
\begin{aligned}
& \begin{array}{lllll}
0 & 4 & 4 & 4 & 4
\end{array} \\
& \begin{array}{lllll}
0 & 0 & 0 & 0 & 0
\end{array} \\
& \begin{array}{llllll}
0 & 0 & 0.1 & 0.5 & 2 & \mathrm{pmol}
\end{array}
\end{aligned}
$$

C
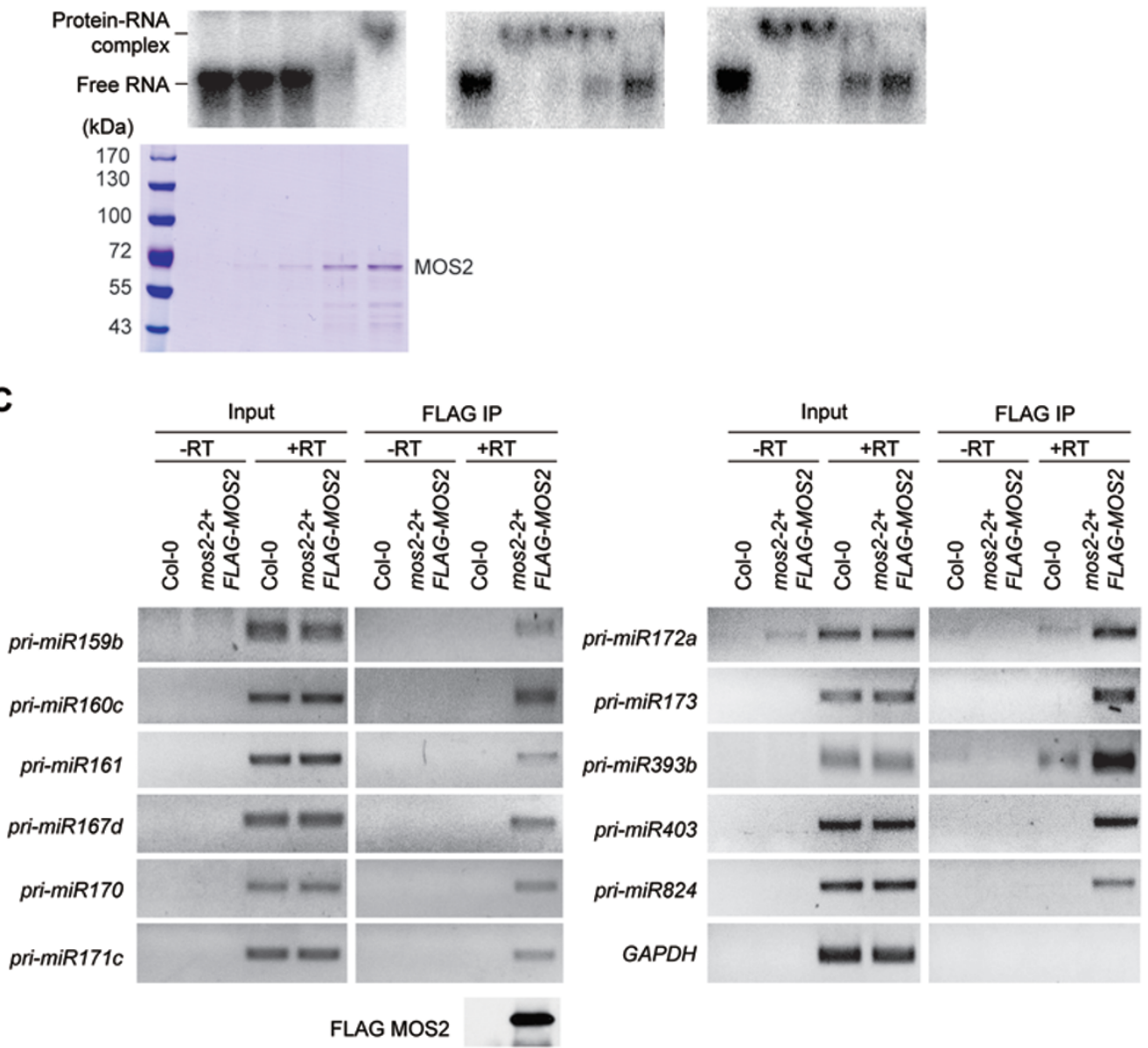

Figure 5 MOS2 binds to pri-miRNAs in vitro and in vivo. (A) Primary sequence and secondary structure of pri-miR159b used in EMSA assays. The miRNA and miRNA* sequences are highlighted in red and blue, respectively. (B) EMSAs were carried out with 0.5 pmol of labeled pri-miR $159 \mathrm{~b}$ transcripts and increasing amounts (0.5-4 pmols) of MOS2 recombinant proteins (left panel). A Coomassie staining gel is shown to indicate the amount and purity of MOS2. In the competition experiments, four pmols of MOS2, 0.5 pmol of labeled pri-miR159b, and increasing amounts $(0.1$ to 2 pmols) of unlabeled pri-miR159b (middle panel) and GAPDH (right panel) transcripts were added in the reactions. (C) Cross-linked nuclear extracts from Col-0 and FLAG-MOS2 transgenic plants were immunoprecipitated with anti-FLAG antibody. RNAs were isolated from the nuclear extracts and immunoprecipitates and analyzed by semi-quantitative RT-PCR using specific primers to detect the indicated primiRNAs. FLAG-MOS2 was detected by immunoblot using anti-MOS2 antibody.

tating the recruitment of pri-miRNAs into the Dicing complex. In order to test this possibility, we examined whether the mos2-2 mutation could affect the binding of pri-miRNAs to HYL1. We crossed pHYL1::HYL1YFP into the mos2-2 mutant. The amount of HYL1-YFP in mos2-2 was comparable to that in Col-0 (Figure 7A), indicating that $\operatorname{mos} 2-2$ did not affect the accumulation of HYL1-YFP. We immunoprecipitated HYL1-YFP complexes from cross-linked seedlings of the transgenic plants in the Col-0 and mos 2-2 mutant backgrounds, re- 

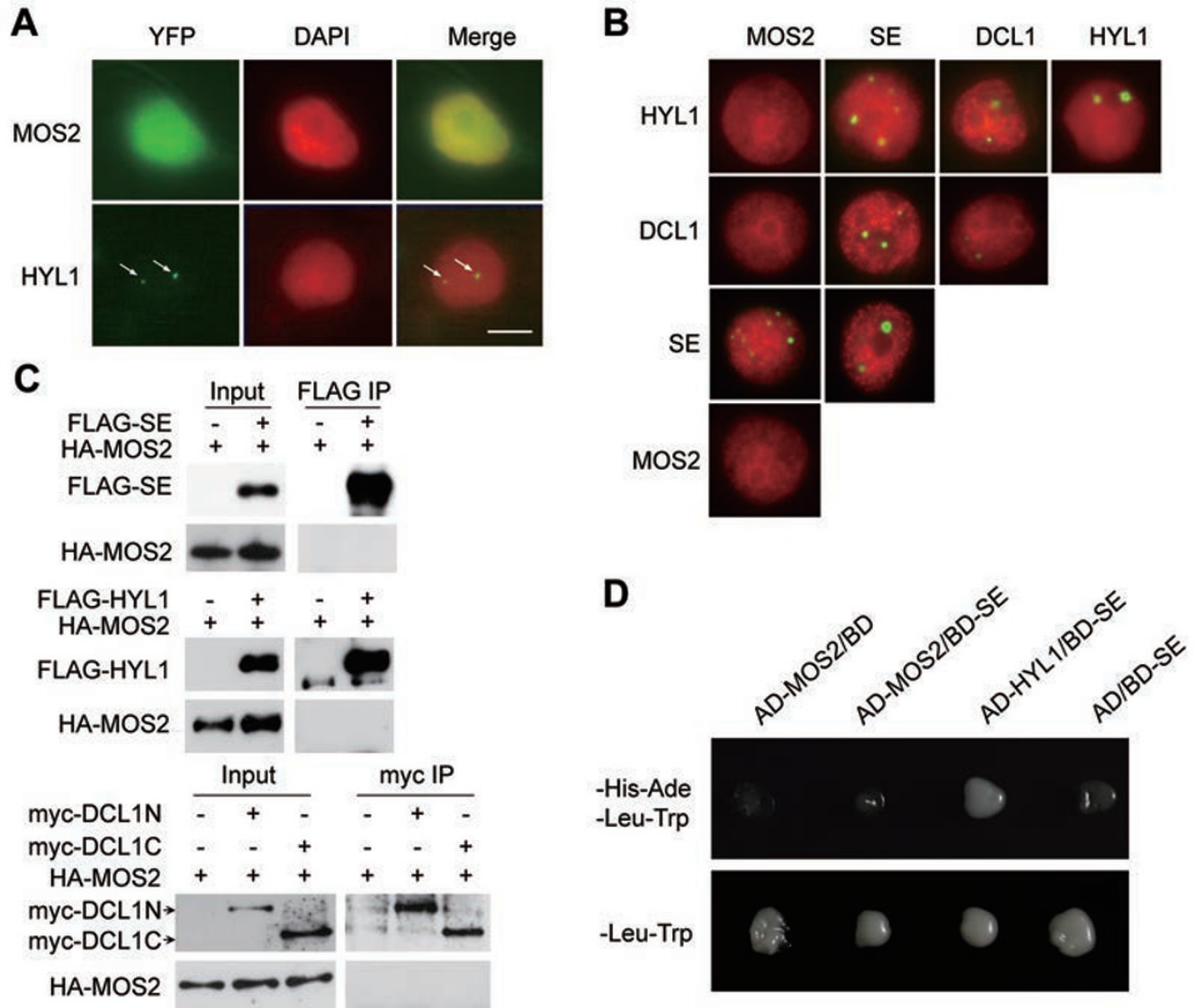

Figure 6 MOS2 does not interact with known protein components in D-bodies. (A) Localization of MOS2-YFP and HYL1YFP in $N$. benthaniana cells. $N$. benthamiana leaves were agroinfiltrated with pMOS2::MOS2-YFP and $p H Y L 1:: H Y L 1-Y F P$ constructs. YFP fluorescence was observed two days post agroinfiltration. Nuclei were labeled with DAPI and pseudocolored in red. Bar $=5 \mu \mathrm{m}$. (B) Pair-wise BiFC experiments between MOS2, SE, DCL1 and HYL1. Protein partners were fused to an $\mathrm{N}$-terminal fragment or C-terminal fragment of YFP, respectively, and co-infiltrated into $\mathrm{N}$. benthaniana leaves. Nuclei were labeled with DAPI and pseudocolored in red. Bar $=5 \mu \mathrm{m}$. (C) Arabidopsis protoplasts were transfected with indicated combinations of constructs. FLAG-SE, FLAG-HYL1, myc-DCL1N, and myc-DCL1C were immunoprecipitated with anti-FLAG or antimyc antibodies. The pull-down products were analyzed by immunoblots with anti-FLAG, anti-myc, or anti-HA antibodies as indicated. (D) Interactions between SE, MOS2 and HYL1 detected by yeast two-hybrid assays. The indicated constructs were transformed to the yeast AH109 strain. Growth on selective medium lacking histidine, adenine, leucine, and tryptophan (-HisAde-Leu-Trp) or on control medium lacking only leucine and tryptophan (-Leu-Trp) is shown.

spectively. Ten pri-miRNAs were detected in the HYL1YFP immonuprecipitates isolated from the transgenic plants in Col-0 background (Figure 7B). However, the amounts of the pri-miRNAs were greatly reduced in the immunoprecipitates prepared from the mos2-2 HYL1YFP plants (Figure 7B), indicating that MOS2 is required for efficient recruitment of pri-miRNAs by HYL1.

We next examined whether the mos 2-2 mutation could affect the localization of HYL1 in D-bodies. In the wild-type plants, D-bodies were detected in nearly all the cells (Figure 7C). However, intriguingly, only $\sim 20 \%$ of cells contained HYL-YFP positive D-bodies in the mos 2-2 mutant (Figure 7C), demonstrating that MOS2 is required for the localization of HYL1 in D-bodies.

It was possible that the failure of HYL1 localization in D-bodies in mos 2-2 was caused by disruption of interactions between the Dicing complex components. To test this possibility, we examined the effect of the mos 2-2 mutation on the interactions between SE, HYL1, and DCL1. Epitope-tagged SE, HYL1, and N-terminal and C-terminal fragments of DCL1 were transiently expressed in wild-type and $\operatorname{mos} 2-2$ protoplasts, and the tagged proteins were immunoprecipitated using epitopespecific antibodies. Confirming published results [4, 3638], interactions between DCL1, HYL1, and SE were detected in wild-type cells (Supplementary information, 
Figure S3), and such interactions were not disrupted in mos 2-2 cells (Supplementary information, Figure S3), indicating that MOS2 is not required for the interactions between these proteins.

\section{Discussion}

Efficient processing of mature miRNAs from pri-miRNAs requires the activity of Dicing complexes comprising DCL1, SE, and HYL1 [29]. It is not clear how Dicing complexes are assembled and how they access primiRNAs. In this study, we identified the RNA-binding protein MOS2 as a novel component in the miRNA pathway. We showed that MOS2 could bind to pri-miRNAs (Figure 5) and was required for their efficient processing (Figure 4). Intriguingly, we observed that the localization of HYL1 in D-bodies was impaired in the mos2-2 mutant (Figure 7). Several possibilities can be considered to interpret this observation: 1) The formation of D-bodies is impaired, 2) D-bodies can be formed but the association of HYL1 with D-bodies is compromised in mos 2-2 cells, and 3) mos 2 mutation might change the nuclear architecture and thereby affect the visible phenotype of D-bodies. We favor the first possibility, given that the interactions
A

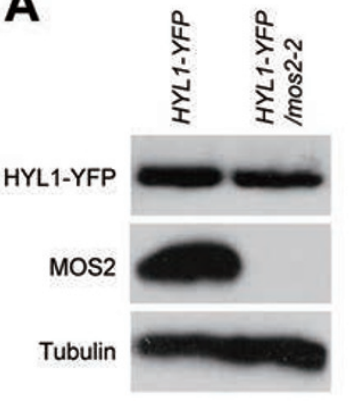

B

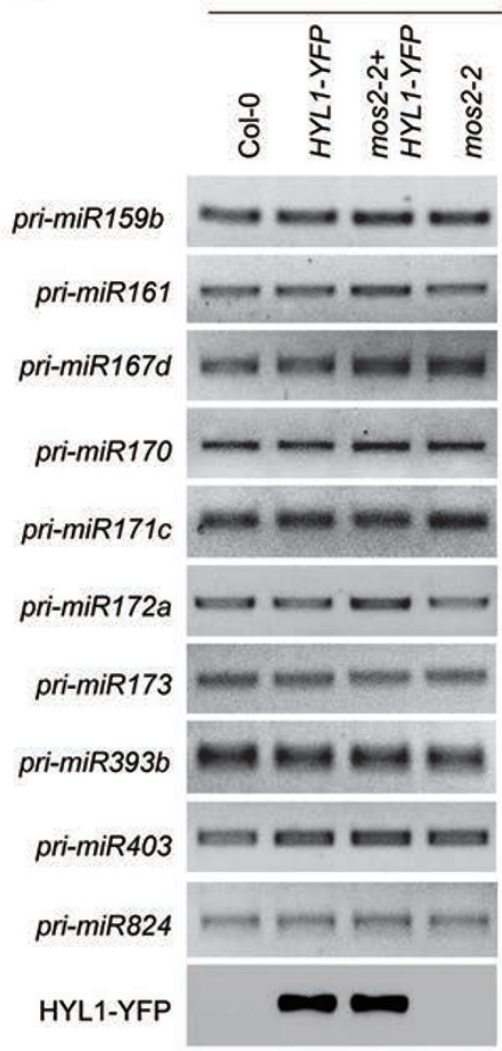

GFP IP

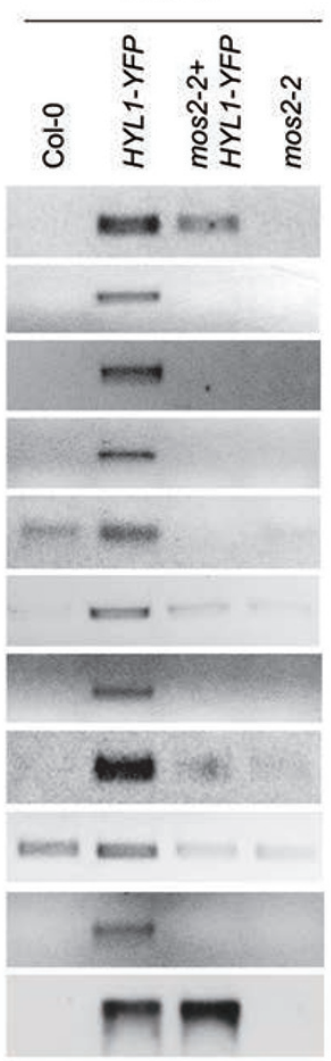

C
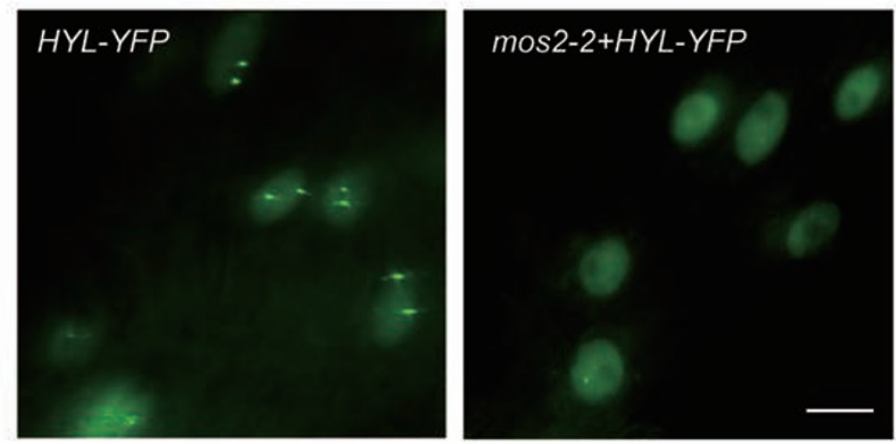

Figure 7 MOS2 facilitates the recruitment of pri-miRNAs by HYL1 and the localization of HYL1 in D-bodies. (A) HYL1YFP and MOS2 protein levels in 2-week-old seedlings of the indicated plants, as measured by immunoblot using YFP and MOS2 antibodies, respectively. Tubulin was also probed and served as the loading control. (B) HYL1-YFP was immunoprecipitated from the 2-weekold seedlings of the indicated plants using a GFP-specific antibody. RNAs were isolated from the nuclear extracts and immunoprecipitates and analyzed by RT-PCR with specific primers to detect the indicated pri-miRNAs. The HYL1-YFP protein was detected by antiGFP antibody. (C) Images of nuclei in root cells of 2-weekold seedlings of the indicated plants. HYL1-YFP was localized in D-bodies in $~ 95 \%$ of the Col- 0 cells and it became evenly distributed in the nucleoplasm in $\sim 80 \%$ of the mos2-2 cells. The percentage of D-body positive nuclei was calculated based on more than 500 cells from 10 plants for each genotype. The error bars indicate SD $(n=500)$, and the asterisk indicates a significant difference between the samples ( $t$ test, $P<0.05$ ). White bar $=5 \mu \mathrm{m}$. 
between HYL1, SE, and DCL1 remained unchanged in mos2-2 (Supplementary information, Figure S3) and that the association between pri-miRNAs and HYL1 was greatly reduced in $\operatorname{mos} 2-2$ (Figure 7 ). We propose that MOS2 promotes pri-miRNA processing through facilitating the recruitment of pri-miRNAs by the Dicing complexes and that pri-miRNAs might function as scaffold transcripts for D-body formation.

MOS2 was first identified in a genetic screen for genes that are involved in disease resistance in Arabidopsis [31]. MOS2 plays a role in innate immunity and the $\operatorname{mos} 2 \mathrm{mu}-$ tants display higher susceptibility to bacterial infection [31]. However, the molecular mechanism through which MOS2 confers antibacterial resistance remains elusive. In this study, we demonstrated a role for MOS2 in the miRNA pathway. In the mos $2-2$ mutant, the accumulation of miRNAs including miR393 was greatly reduced (Figure 1B), which was accompanied by increased accumulation of their target mRNAs (Figure 2B). It has been previously shown that miR393 contributes to antibacterial resistance through repressing its target genes that encode the F-box auxin receptors TIR1, AFB2, and AFB3 [39]. Several other miRNAs have also been found to be involved in antibacterial resistance [40]. Thus, MOS2 modulates innate immunity at least partly through its function in the miRNA pathway.

In addition to its role in miRNA biogenesis, we found MOS2 is also involved in the biogenesis of ta-siRNAs and hc-siRNAs (Figure 1B). It is currently unknown how MOS2 functions in these siRNA pathways. As the biogenesis of ta-siRNAs is dependent on the miRNAmediated cleavage of their precursor transcripts, the role of MOS2 in ta-siRNA biogenesis might be indirect. However, it has been reported that HYL1 and its homolog DRB4 are co-localized in D-bodies [16]. DRB4 interacts with DCL4 and is involved in the biogenesis of ta-siRNAs [41-43]. Thus, it remains possible that MOS2 functions in ta-siRNA biogenesis through regulating the localization of DRB4 in D-bodies. Two other RNA binding proteins, DDL [10] and TOUGH [44], have also been shown to be involved in the biogenesis of both miRNAs and siRNAs. It is likely that these RNA-binding proteins have broader functions in RNA metabolism. A link between RNA metabolism and silencing has been reported [7-9].

MOS2 has homologs in human, mouse, and worm [31, 45]. A genome-wide RNAi screen in worm indicated that knock-down of the MOS2 homolog causes embryonic lethality [46]. In light of our finding that MOS2 is required for miRNA maturation, it is possible that this embryonic lethality is caused by a defect in miRNA biogenesis in worm. It will be of great interest to examine whether the
MOS2 homologs play a role in miRNA biogenesis in these organisms.

\section{Materials and Methods}

\section{Plant materials and growth conditions}

The Arabidopsis thaliana lines used in this study are all in the Columbia (Col-0) background. mos2-2 is a T-DNA insertion line (SALK 033856) described previously [31]. hyll-2 is a T-DNA insertion line (SALK_064683) as described [12] and ago1-25 is a hypomorphic allele of AGO1 [33]. The transgenic lines pHYL1::HYL1-YFP [16], pMIR159b::GUS [34] and pMIR398c::GUS [35] were previously described. Transgenic plants were constructed using a floral dip method [47]. Positive transformants were selected by antibiotic markers and confirmed by western blot. All plants were grown at 16 -h-light/8-h-dark or 10-h-light/14-h-dark (for protoplast isolation) photoperiod.

\section{DNA constructs}

BiFC constructs $p D C L 1: \because D C L 1-Y F P N, p D C L 1:: D C L 1-Y F P C$, pHYL1::HYL1-YFPN, pHYL1::HYL1-YFPC, pSE::YFPN-SE, and $p S E:: Y F P C-S E$ have been described previously [16]. To construct BiFC constructs of MOS2, the genomic sequence of MOS 2 including its promoter was amplified from Arabidopsis genomic DNA using gMOS2-F and gMOS2-R primers (Supplementary information, Table S3), digested with BamHI and SalI, and then cloned into BamHI/SalI-treated $p$ Cambia1300-N1-YFPN and $p C a m-$ bia1300-N1-YFPC vectors [16], respectively, resulting in pMOS2$M O S 2-Y F P N$ and $p M O S 2-M O S 2-Y F P C$. The same genomic fragment was also cloned into $p$ Cambia1300-N1-YFP, to generate pMOS2::MOS2-YFP.

cDNA fragments of $M O S 2, H Y L 1$, and $S E$ were amplified by RT-PCR using primers listed in Supplementary information, Table S3, and inserted between BamHI and SalI sites in modified pCambia1307 vectors containing the $3 \times$ FLAG or $3 \times \mathrm{HA}$ coding sequence, to obtain $p 35 S: \because F L A G-M O S 2$, p35S::FLAG$S E$, p35S::HA-MOS2, and p35S::FLAG-HYL1 constructs. The Nterminal of $D C L 1$ or the C-terminal of $D C L 1$ was amplified using primers DCL1N-F and DCL1N-R or DCL1C-F and DCL1C-R, and then cloned into $\mathrm{pENTR/D-TOPO}$ (Invitrogen) to generate pEDCL1N or pEDCL1C. The DCL1N or DCL1C fragment was transferred from $\mathrm{pEDCL} 1 \mathrm{~N}$ or pEDCL1C into pEarleyGate203 vector through LR recombination (Invitrogen), resulting in p35S: $m y c-D C L 1 N$ and $p 35 S$ : $m y c-D C L 1 C$. The MOS2 cDNA fragment was also cloned into a modified pET28a vector with an amino-terminal hexahistidine-SUMO-tag, resulting in pET28a:MOS2 construct for expression in E. coli. Oligonucleotide primers are listed in Supplementary information, Table S3.

To construct the vectors for yeast two-hybrid assay, MOS2, $S E$ and HYL1 fragments were cloned into pENTR/D-TOPO (Invitrogen) and subsequently transferred to the destination vectors pDEST-GADT7 and pDEST-GBKT7 through LR recombination.

\section{Small RNA northern blot}

Small RNA northern blot analysis with enriched small RNAs was performed as described [22]. ${ }^{32} \mathrm{P}$-end-labeled oligonucleotides complementary to miRNA, ta-siRNA, or hc-siRNA sequences were used as probes. The sequences of the probes are listed in 
Supplementary information, Table S3.

\section{$m R N A$ sequencing analysis}

Total RNAs were isolated from two-week-old seedlings of Col0 and mos 2-2 using TRizol (Invitrogen) and mRNAs were purified using Oligotex mRNA Mini Kit (Qiagen). cDNA libraries were generated for Illumina GAIIx platform according to the manufacturer's instructions (Illumina). Raw reads from Illumina sequencing were mapped to the Arabidopsis genome using TopHat [48]. Cuffdiff [49] was used for expression level estimation (given in FPKM, Fragments Per Kilobase of transcript per Million mapped reads) and differential expression analysis (measured by $q$-value, multiple testing correction of $P$-value) with default parameters. Transcripts were considered as differentially expressed when they satisfied the criteria: fold-change $\geq 2$ and $q$-value $<0.05$.

\section{Quantitative RT-PCR}

Total RNA was extracted with the Trizol reagent (Invitrogen) from 18-d-old soil-grown plants. Total RNA was treated with RNase-free DNase I (Promega) to remove DNA, and reversetranscribed by M-MLV reverse transcriptase (Promega) using oligo(dT). Quantitative PCR was performed with SYBR Premix EX Taq (TAKARA). GAPDH mRNA was detected in parallel and used for data normalization. The primers used for PCR are listed in Supplementary information, Table S3.

\section{GUS staining}

Plant materials were infiltrated with $50 \mathrm{mM}$ sodium phosphate (pH 7.0), $10 \mathrm{mM}$ EDTA, and $0.5 \mathrm{mg} / \mathrm{ml} \mathrm{X-gluc} \mathrm{(Apollo} \mathrm{Scientif-}$ ic), followed by incubation at $37^{\circ} \mathrm{C}$ in dark overnight. Then plantlets were cleared in ethanol and the blue sectors in each plantlet were counted under a stereomicroscope (Nikon) and representative pictures were taken.

\section{Preparation of recombinant MOS2}

pET28a:MOS2-containing E.coli Rossetta2 cells were grown to an OD600 of $\sim 0.6$ and induced for $16 \mathrm{~h}$ at $18{ }^{\circ} \mathrm{C}$ using $0.5 \mathrm{mM}$ isopropyl $\beta$-d-thiogalactoside (IPTG). Cells were harvested and resuspended in buffer A (50 mM Tris- $\mathrm{HCl}, \mathrm{pH} 8.0,150 \mathrm{mM} \mathrm{NaCl}$, $5 \%$ glycerol and $1 \mathrm{mM}$ PMSF), then lysed by sonication. The nHis-SUMO-fused MOS2 was purified using a histrap FT column (GE). The nHis-SUMO tag in the recombinant protein was cleaved by ULP1 enzyme and removed by reloading onto a histrap column. The resulting non-tagged MOS2 protein was further purified by a Superdex 200 column (GE). Purified MOS2 proteins were used for raising polyclonal antibodies and electrophoretic mobility shift assay (EMSA).

\section{Electrophoretic mobility shift assay (EMSA)}

The in vitro RNA-binding activity of MOS2 was assayed by EMSA experiments. Pri-miR159b and GAPDH fragments were amplified from Col-0 cDNAs by PCR using specific primers with a $\mathrm{T} 7$ promoter sequence fused to the $5^{\prime}$ termini of the forward primers (Supplementary formation, Table S1). The PCR products were used as templates for in vitro transcription (Promega) to make ${ }^{32} \mathrm{P}$ radiolabeled or unlabeled RNA transcripts. EMSA experiments were performed in a $10-\mu 1$ reaction mixture containing $10 \mathrm{mM}$ Tris- $\mathrm{HCl}, \mathrm{pH} 7.5,100 \mathrm{mM} \mathrm{NaCl}, 2 \mathrm{mM} \mathrm{MgCl}_{2}, 0.2 \mathrm{mM}$ EDTA, $1 \mathrm{mM}$ DTT, $0.5 \%$ bovine serum albumin, $5 \%$ glycerol, $0.01 \%$
NP-40 and 40 unit RNase Inhibitor (Promega) and 0.5 pmol of labeled pri-miR159b transcripts and increasing amounts of MOS2 recombinant proteins. The mixtures were incubated on ice for 30 min and then electrophoresed on a 5\% native polyacrylamide gel in $1 \times$ Tris-glycine buffer $(\mathrm{pH} 8.0)$ for about $30 \mathrm{~min}$. The gels were dried and autoradiographed. In the competition experiments, four pmols of MOS2, 0.5 pmol of labeled pri-miR159b, and increasing amounts ( 0.1 to 2 pmols) of unlabeled pri-miR159b and GAPDH transcripts were added in the reactions.

\section{Agroinfiltration and bimolecular fluorescence complemen- tation (BiFC) experiments}

Agroinfiltration and $\mathrm{BiFC}$ experiments were performed essentially as described [16] except that $N$. benthamiana plants were used.

\section{Yeast two-hybrid assay}

Yeast two-hybrid assay was performed using the Matchmaker GAL4 two-hybrid system (Clontech), according to the manufacturer's instructions.

\section{Fluorescence microscopy}

Images of the nuclei in the agroinfiltrated $N$. benthamiana cells were acquired with a fluorescence imaging workstation consisted of an TE2000-E microscope (Nikon) equipped with a Plan-Apochromat VC 100× 1.4 NA objective (Nikon Instruments), a charge coupled device (CCD) camera(DS-2MBWc, Nikon) and filters for YFP, exciter, $500 / 20 \mathrm{~nm} / \mathrm{nm}$; emitter, $535 / 30 \mathrm{~nm} / \mathrm{nm}$; for DAPI, exciter, $360 / 40 \mathrm{~nm} / \mathrm{nm}$; emitter, $450 / 60 \mathrm{~nm} / \mathrm{nm}$.

For fluorescence microscopy analysis of the transgenic plants, roots of ten-day-old seedlings were treated with DAPI (Sigma) and analyzed using an EC plan-NEOFLUAR 100× oil dipping objective lens $(100 \times$, numerical aperture 1.3 ; Zeiss) on a Zeiss Imager M1 AX10 microscope. Images were captured with a Zeiss Axiocam HRc CCD camera and processed using AxioVision software (Zeiss). Images in the YFP and DAPI channels were acquired. Filters used were as follows: for YFP, exciter, 488/512 nm/nm; emitter, $520 / 50 \mathrm{~nm} / \mathrm{nm}$; for DAPI, exciter, $335 / 83 \mathrm{~nm} / \mathrm{nm}$; emitter, $420 / 70 \mathrm{~nm} / \mathrm{nm}$.

\section{Protoplast transformation}

Protoplast preparation and transfection were performed as described [50].

\section{Immunoprecipitation}

Immunoprecipitation of epitope-tagged proteins from transfected Arabidopsis protoplasts was performed as described [28] using commercial antibodies recognizing the corresponding epitopes.

\section{Western blot}

Plant protein extracts or immunoprecipitates were loaded on an $8 \%$ SDS-PAGE gel for protein separation. After transferred to PVDF membranes, proteins were detected using homemade or commercial antibodies.

\section{RNA immunoprecipitation}

RNA immunoprecipitation was carried out essentially as described [51] using 1\% formaldehyde treated three-week-old seedlings. Pri-miRNAs were detected in the immunoprecipitates by 
RT-PCR using primers listed in Supplementary information, Table S1. The positions of the primers in the pri-miRNA sequences are shown in Supplementary information, Figure S4.

\section{Accession numbers}

RNA-seq datasets from this article can be found in the NCBI Gene Expression Omnibus (http://www.ncbi.nlm.nih.gov/geo/) under accession number GSE41843.

\section{Acknowledgments}

We thank Dr Anthony Millar (The Australian National University, Australian) for pMIR159b::GUS, Dr Bonnie Bartel (Rice University, USA) for $p M I R 398 c:: G U S$ and Dr H Vaucheret (Institut National de la Recherche Agronomique, France) for the hyl1-2 and ago1-25 mutants. This work was supported in part by China National Funds for Distinguished Young Scientists (31225015) and National Basic Research Program of China (2012CB910900) to Y Q.

\section{References}

1 Carthew RW, Sontheimer EJ. Origins and mechanisms of miRNAs and siRNAs. Cell 2009; 136:642-655.

2 Kim VN. MicroRNA biogenesis: coordinated cropping and dicing. Nat Rev Mol Cell Biol 2005; 6:376-385.

3 Kurihara Y, Watanabe Y. Arabidopsis micro-RNA biogenesis through Dicer-like 1 protein functions. Proc Natl Acad Sci USA 2004; 101:12753-12758.

4 Kurihara Y, Takashi Y, Watanabe Y. The interaction between DCL1 and HYL1 is important for efficient and precise processing of pri-miRNA in plant microRNA biogenesis. $R N A$ 2006; 12:206-212.

5 Park W, Li J, Song R, Messing J, Chen X. CARPEL FACTORY, a Dicer homolog, and HEN1, a novel protein, act in microRNA metabolism in Arabidopsis thaliana. Curr Biol 2002; 12:1484-1495.

6 Reinhart BJ, Weinstein EG, Rhoades MW, Bartel B, Bartel DP. MicroRNAs in plants. Genes Dev 2002; 16:1616-1626.

7 Laubinger S, Sachsenberg T, Zeller G, et al. Dual roles of the nuclear cap-binding complex and SERRATE in pre-mRNA splicing and microRNA processing in Arabidopsis thaliana. Proc Natl Acad Sci USA 2008; 105:8795-8800.

8 Kim S, Yang JY, Xu J, Jang IC, Prigge MJ, Chua NH. Two cap-binding proteins CBP20 and CBP80 are involved in processing primary MicroRNAs. Plant Cell Physiol 2008; 49:1634-1644.

9 Gregory BD, O'Malley RC, Lister R, et al. A link between RNA metabolism and silencing affecting Arabidopsis development. Dev Cell 2008; 14:854-866.

$10 \mathrm{Yu}$ B, Bi L, Zheng B, et al. The FHA domain proteins DAWDLE in Arabidopsis and SNIP1 in humans act in small RNA biogenesis. Proc Natl Acad Sci USA 2008; 105:10073-10078.

11 Han MH, Goud S, Song L, Fedoroff N. The Arabidopsis double-stranded RNA-binding protein HYL1 plays a role in microRNA-mediated gene regulation. Proc Natl Acad Sci USA 2004; 101:1093-1098.

12 Vazquez F, Gasciolli V, Crete P, Vaucheret H. The nuclear dsRNA binding protein HYL1 is required for microRNA ac- cumulation and plant development, but not posttranscriptional transgene silencing. Curr Biol 2004; 14:346-351.

13 Grigg SP, Canales C, Hay A, Tsiantis M. SERRATE coordinates shoot meristem function and leaf axial patterning in Arabidopsis. Nature 2005; 437:1022-1026.

14 Lobbes D, Rallapalli G, Schmidt DD, Martin C, Clarke J. SERRATE: a new player on the plant microRNA scene. EMBO Rep 2006; 7:1052-1058.

15 Song L, Han MH, Lesicka J, Fedoroff N. Arabidopsis primary microRNA processing proteins HYL1 and DCL1 define a nuclear body distinct from the Cajal body. Proc Natl Acad Sci USA 2007; 104:5437-5442.

16 Fang Y, Spector DL. Identification of nuclear dicing bodies containing proteins for microRNA biogenesis in living Arabidopsis plants. Curr Biol 2007; 17:818-823.

17 Dong Z, Han MH, Fedoroff N. The RNA-binding proteins HYL1 and SE promote accurate in vitro processing of primiRNA by DCL1. Proc Natl Acad Sci USA 2008; 105:99709975.

18 Manavella PA, Hagmann J, Ott F, et al. Fast-forward genetics identifies plant CPL phosphatases as regulators of miRNA processing factor HYL1. Cell 2012; 151:859-870.

19 Li J, Yang Z, Yu B, Liu J, Chen X. Methylation protects miRNAs and siRNAs from a 3'-end uridylation activity in Arabidopsis. Curr Biol 2005; 15:1501-1507.

20 Yu B, Yang Z, Li J, et al. Methylation as a crucial step in plant microRNA biogenesis. Science 2005; 307:932-935.

21 Mi S, Cai T, Hu Y, et al. Sorting of small RNAs into Arabidopsis argonaute complexes is directed by the 5 ' terminal nucleotide. Cell 2008; 133:116-127.

22 Qi Y, Denli AM, Hannon GJ. Biochemical specialization within Arabidopsis RNA silencing pathways. Mol Cell 2005; 19:421-428.

23 Baumberger N, Baulcombe DC. Arabidopsis ARGONAUTE1 is an RNA Slicer that selectively recruits microRNAs and short interfering RNAs. Proc Natl Acad Sci USA 2005; 102:11928-11933.

24 Vaucheret H, Vazquez F, Crete P, Bartel DP. The action of ARGONAUTE1 in the miRNA pathway and its regulation by the miRNA pathway are crucial for plant development. Genes Dev 2004; 18:1187-1197.

25 Iki T, Yoshikawa M, Meshi T, Ishikawa M. Cyclophilin 40 facilitates HSP90-mediated RISC assembly in plants. EMBO $J$ 2011; 31:267-278.

26 Iki T, Yoshikawa M, Nishikiori M, et al. In vitro assembly of plant RNA-induced silencing complexes facilitated by molecular chaperone HSP90. Mol Cell 2010; 39:282-291.

27 Smith MR, Willmann MR, Wu G, et al. Cyclophilin 40 is required for microRNA activity in Arabidopsis. Proc Natl Acad Sci USA 2009; 106:5424-5429.

28 Wang $\mathrm{W}$, Ye R, Xin Y et al. An importin $\beta$ protein negatively regulates microRNA activity in Arabidopsis. Plant Cell 2011; 23:3565-3576.

29 Voinnet O. Origin, biogenesis, and activity of plant microRNAs. Cell 2009; 136:669-687.

$30 \mathrm{Wu}$ L, Zhou H, Zhang Q, et al. DNA methylation mediated by a microRNA pathway. Mol Cell 2010; 38:465-475.

31 Zhang Y, Cheng YT, Bi D, Palma K, Li X. MOS2, a protein containing G-patch and KOW motifs, is essential for innate 
immunity in Arabidopsis thaliana. Curr Biol 2005; 15:19361942.

32 Baulcombe D. RNA silencing in plants. Nature 2004; 431:356-363.

33 Morel JB, Godon C, Mourrain P, et al. Fertile hypomorphic ARGONAUTE (ago1) mutants impaired in post-transcriptional gene silencing and virus resistance. Plant Cell 2002; 14:629-639.

34 Allen RS, Li J, Stahle MI, Dubroué A, Gubler F, Millar AA. Genetic analysis reveals functional redundancy and the major target genes of the Arabidopsis miR159 family. Proc Natl Acad Sci USA 2007; 104:16371-16376.

35 Dugas DV, Bartel B. Sucrose induction of Arabidopsis miR398 represses two $\mathrm{Cu} / \mathrm{Zn}$ superoxide dismutases. Plant Mol Biol 2008; 67:403-417.

36 Yang L, Liu Z, Lu F, Dong A, Huang H. SERRATE is a novel nuclear regulator in primary microRNA processing in Arabidopsis. Plant J 2006; 47:841-850.

37 Hiraguri A, Itoh R, Kondo N, et al. Specific interactions between Dicer-like proteins and HYL1/DRB-family dsRNAbinding proteins in Arabidopsis thaliana. Plant Mol Biol 2005; 57:173-188.

38 Yang SW, Chen HY, Yang J, Machida S, Chua NH, Yuan YA. Structure of Arabidopsis HYPONASTIC LEAVES1 and its molecular implications for miRNA processing. Structure 2010; 18:594-605.

39 Navarro L, Dunoyer P, Jay F, et al. A plant miRNA contributes to antibacterial resistance by repressing auxin signaling. Science 2006; 312:436-439.

40 Li Y, Zhang Q, Zhang J, Wu L, Qi Y, Zhou JM. Identification of microRNAs involved in pathogen-associated molecular pattern-triggered plant innate immunity. Plant Physiol 2010; 152:2222-2231.

41 Fukudome A, Kanaya A, Egami M, et al. Specific requirement of DRB4, a dsRNA-binding protein, for the in vitro dsRNAcleaving activity of Arabidopsis Dicer-like 4. RNA 2011;
17:750-760.

42 Nakazawa Y, Hiraguri A, Moriyama H, Fukuhara T. The dsRNA-binding protein DRB4 interacts with the Dicer-like protein DCL4 in vivo and functions in the trans-acting siRNA pathway. Plant Mol Biol 2007; 63:777-785.

43 Adenot X, Elmayan T, Lauressergues D, et al. DRB4-dependent TAS3 trans-acting siRNAs control leaf morphology through AGO7. Curr Biol 2006; 16:927-932.

44 Ren G, Xie M, Dou Y, Zhang S, Zhang C, Yu B. Regulation of miRNA abundance by RNA binding protein TOUGH in Arabidopsis. Proc Natl Acad Sci USA 2012; 109:12817-12821.

45 Aksaas AK, Larsen AC, Rogne M, Rosendal K, Kvissel AK, Skålhegg BS. G-patch domain and KOW motifs-containing protein, GPKOW; a nuclear RNA-binding protein regulated by protein kinase A. J Mol Signal 2011; 6:10.

46 Piano F, Schetter AJ, Morton DG, et al. Gene clustering based on RNAi phenotypes of ovary-enriched genes in C. elegans. Curr Biol 2002; 12:1959-1964.

47 Clough SJ, Bent AF. Floral dip: a simplified method for Agrobacterium-mediated transformation of Arabidopsis thaliana. Plant J 1998; 16:735-743.

48 Trapnell C, Pachter L, Salzberg SL. TopHat: discovering splice junctions with RNA-Seq. Bioinformatics 2009; 25:1105-1111.

49 Trapnell C, Williams BA, Pertea G, et al. Transcript assembly and quantification by RNA-Seq reveals unannotated transcripts and isoform switching during cell differentiation. Nat Biotechnol 2010; 28:511-515.

50 Yoo SD, Cho YH, Sheen J. Arabidopsis mesophyll protoplasts: a versatile cell system for transient gene expression analysis. Nat Protoc 2007; 2:1565-1572.

51 Wierzbicki AT, Haag JR, Pikaard CS. Noncoding transcription by RNA polymerase Pol IVb/Pol V mediates transcriptional silencing of overlapping and adjacent genes. Cell 2008; 135:635-648.

(Supplementary information is linked to the online version of the paper on the Cell Research website.) 